\title{
Evaluation of an integrated sensory glove at decreasing joint flexion degree
}

\author{
Giovanni Saggio, Giancarlo Orengo, Antonio Pallotti, Vito Errico, Mariachiara Ricci \\ Department of Electronic Engineering \\ University of Rome "Tor Vergata", Rome, Italy
}

\begin{abstract}
The glove remains the main tool for detecting the flexion angles of the hand joints. Semiautomatic measurements of the hand joints are carried out to date with the use of the goniometric glove. This study describes a new data glove equipped with 10 bending sensors, showing results similar to those already existing in the literature. An automatic measurement system has been developed, to analyze the nonlinear behavior of flex sensors through the measurement of the bending angles between hand phalanges. This paper suggests the study of two intermediate joint flexion degree between the open and closed hand, to analyze the non-linearity of sensors in the Wise protocol, extending it. The Wise protocol was applied with two molds to allow two intermediate closure with respect to the open and the closed hand. The dependence of repeatability, reproducibility, reliability and correlation between Range and SD depending on the size of the mold used for the Wise test are also investigated. It has been seen that the size of the mold could influences the Range and SD values.
\end{abstract}

Keywords-Sensory glove; resistive bend sensors; goniometer measurements; wearable device, hand motion capture.

\section{INTRODUCTION}

In physiological conditions, the hands are used to implement the will and allow for sustaining actions such as cooking, eating, grasping, and social interaction through devices such as keyboard writing, touchscreen using, camera gesture recognition or the sign language communication for deaf people. The degree of hand motion is used for assessing the movement disorder and evaluating the effectiveness of a rehabilitation therapy. In the industrial environment, manipulators are driven by an interface that reduces the degrees of freedom of the hand and upper limb. The same limitations are found in surgical, spatial and war manipulators. Sensor-based systems for detecting, recognizing and measuring the position and movement of the fingers have been widespread in recent years. The performance of sensory gloves did not increase at the same rate of application developed to exploit the potential of these systems. Data gloves equipped with optical [1], magnetic, flex [2,3], acoustic, Hall-effect, IMU sensors [4,5] allow the detection of flexion-extension of the finger joints.

The use of optical systems imposes limitations on affordability for high costs, laboratory space constraints, loss of information when a marker is not detected by a camera. The study of the repeatability, reproducibility, reliability of similar systems was carried out by Wise et al., which considers only two positions in the study under the hypothesis of a linear behavior of the sensors between them $[6,7,8]$. The Wise protocol was used for data gloves of different physical principle. Sensor non-linearity is most evident for low bending angles. Compared to manual measurements performed by therapists, it reached to acceptable range values for flex sensor-based gloves. Combinations of sensors, such as flex and IMU, have been proposed to increase data glove performance [5]. Some gloves reach acceptable range values while others have not been studied through the Wise test. A combination of IMU and flex sensor based glove has been proposed for application to real time translate of Italian Sign Language [9].

\section{MATERIALS}

A wireless sensor system was used with an antenna and data storage software to perform the study. It has been used: CAD software (Solidworks) and 3D printing software (Makerware), 3D printer (Makerbot Replicator 2) with PLA filament, electronic board such as Arduino Nano and Arduino IDE for firmware programming, led and speaker for hand positioning pacemaker. Six right-hand volunteers were employed for the Wise test.

\section{A. Sensory glove}

The HiTEG glove has 10 flexion-extension sensors located at the proximal metacarpophalangeal (MP) and proximal interphalangeal (PIP) joints, except for the thumb that has a proximal IP (PIP) sensor and distal IP sensor (DIP). 10 degrees of freedom are detected corresponding to 4-finger PIP and MP joints and DIP and PIP thumb joints. The sensors are developed by Flexpoint (Flexpoint Sensor Systems) and already used in previous studies [10]. The selected resistive sensors are stable, low cost, with a thickness less than 5 mils, flexible, almost linear. Different sensor sizes can be also selected depending on the application: sensors of 1, 2, 3-inches are available for the finger joints DIP, PIP, MCP, respectively. The influence of fabric composition on sliding and flexion of the sensor has been studied. The Hiteg glove is made of $88 \%$ polyester and $12 \%$ Elastane, because it provides greater comfort for movement. For the present study, only one medium right hand glove was sufficient. Additional gloves have been developed for the left hand and the different sizes (small and large). The selected glove could be best worn by the largest number of participants. The used sensory glove covers all the hand, but it can be modified to let the palmar surface and the 
fingertips free to maintain the tactile sensitivity. The wiring connection of the sensors to the electronic board is flexible and with a diameter of $1.5 \mathrm{~mm}$ and a weight less than $1 \mathrm{~g} / \mathrm{m}$. The sensory glove has a $50 \mathrm{~g}$ weight.

A goniometer has been designed and printed to be applied on the phalanxes with 1 degree sensitivity for the manual measurement of the bending angles. The goniometer has two arms each of the size of a phalanx so that it can adhere to the fingers without any interference from the rest of the hand.

\section{B. Data acquisition and communication}

The Arduino Leonardo electronic board allows the management of 10 analog inputs, 2 digital pin for bluetooth serial communication, and provides ground and voltage reference for operation of the bend sensors. The transduction takes place via a voltage divider between 0 and $5 \mathrm{~V}$ with a fixed $18 \mathrm{~K} \Omega$ resistance, selected because it is the geometric mean between the maximum and minimum sensor resistance, to provide the widest voltage range [11]. The sensor readout circuit is followed by a 10 bit ADC module. The ADC and transmission frequencies are fixed at $50 \mathrm{~Hz}$ and, once the data are acquired, they are redirected to the bluetooth module and sequentially to the receiving antenna connected to a PC. Data missing was not observed during the test. For data transmission on 8 bit serial communication, the 10 bit data from ADC have been decomposed into High and Low byte. Known the high and low part sending sequence, it was able to reconstruct the original given data to 10 bits. To separate the packet sent to $50 \mathrm{~Hz}$, a packet start flag was inserted, so that it could be acknowledged in reception at each loop, thus allowing the separation of the data at each acquisition cycle. The electronic board might work longer for firmware optimization (for example, transmitting only 6 seconds instead of 10 seconds for each position). It is synchronized with the timer of the audio-luminous stimuli to make the times known. The sensory glove can work both alone and within a network of sensors and can be interfaced with mobile devices, smartphones, tablets and cloud data storage systems. The electronic board has a 250 gr. weight, largely due to the Arduino Leonardo board and the $7.4 \mathrm{~V}$ power battery.

Antenna transmission is via bluetooth serial communication. For the transmission is used the RN42 mate silver module for low power consumption: a bluetooth 2.0 dongle is used for the receiving computer.

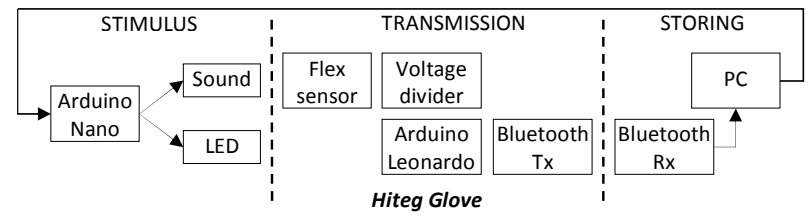

Fig.1. The diagram illustrates the 3 stages of the system. The audio-visual stimulus marks the time and where to place the hand. Synchronously, data are captured and wirelessly transmitted to the receiving antennas connected to the PC. The PC stores the data and redirects the synchronization signal from the acquiring board to the pacing board.

A GUI was developed in the Labview environment for data receiving and storing on pc. Data transmission from the glove board is continuous, once it is powered, whereas start and stop data storing are activated by virtual button. Data are saved in 'txt' format. The developed interface allows the digital values from 10 glove sensors to be visualized and tracked. If necessary, the avatar of the virtual hand may be displayed during recording.

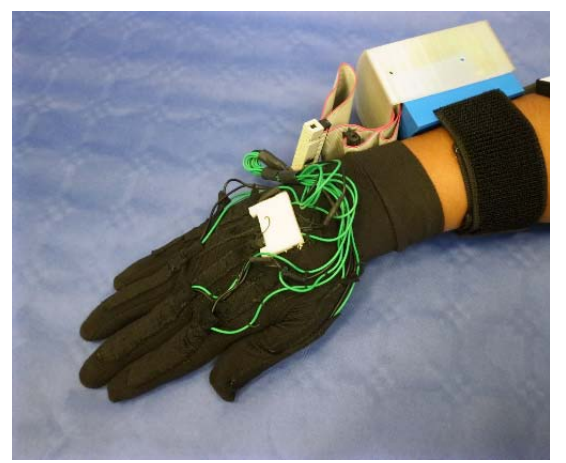

Fig.2. The two sensory unit. The dataglove for the IP and MP hand joints.

Solidworks 2016 has been used for the mold design, which provides input formatting for the $3 \mathrm{D}$ printer software. Makerware allows to select the 3D printing material, PLA in our study, the temperature and displacement velocity of the extruder, the degree of object thickening, the spaces to be left empty within the solid. The Makerbot Replicator 2 printer performs the instructions in the Makerware output file. Molten filament 3D printer takes place in a couple of hours for molds drawn. The large mold (is a cylinder of $63 \mathrm{~mm}$ diameter) has a diameter of $63 \mathrm{~mm}$, and the small mold of 53 $\mathrm{mm}$. These two sizes have been selected because they are between open and closed hand: the mean values measured with the 3D printed goniometer are 30 degrees for MP and PIP with large mold, whereas they are 45 degrees for MP and PIP for small mold.

\section{METHODS}

Six healthy subjects were involved in the test, 4 males and 2 females, all right-handed, 40 years mean age with $\mathrm{SD}= \pm 20$ years. The measurement protocol was approved by the local ethics committee. The subjects were selected so that the glove was worn by a hand of suitable size: male hand fits the glove better than the female one. As reported in $[2,6,7,8]$, the same glove size offers better performance for males than females who tend to have a smaller hand: in the second case the glove performance aren't optimal due to the reduced adhesion and the incorrect positioning of the sensors with respect to the joints to be measured. To perform the test, subjects were selected, as far as possible, which have a comparable hand size. The glove was worn by the right hand and the electronic board was attached to the forearm. The board was activated before being mounted on the forearm. The measuring setup consisted of 4 areas: an area to place 
the open hand at rest and where a reference was given, a large mold area, a small mold area, a closed hand area. The subject was sitting in a chair with his back resting on the backrest, his left hand resting on his left leg and the right hand layed open on the setup with the palm down. The hand positioning during the Wise test is shown in Fig. 3. Before the registration started, iteration tests were performed so that the subject gained confidence in the test; all the sensors were checked to fit the hand and their qualitative tracks were controlled. Maximum and minimum calibration values were provided for the glove before each measurement block: for the minimum value the subject keeps the open hand extending on the table, thumbs at 45 degrees from the index and in rest, for the maximum value the subject closes the hand with force. For the calibration with the molds, the same procedure was used for the minimum, whereas the mold was gripped with force for the maximum. Each position is mantained for a period of 10 seconds. Each period is driven by the Arduino Nano, that emits a musical note and keeps the corresponding led on for each position. The first 40-second iteration cycle includes the static maintenance of the four test positions. 10 seconds of recording were selected from which the 6 central seconds are extracted for the analysis. At the end of the 10 iterations, repeated in 10 blocks (with a break of at least 3 minutes between two consecutive blocks), an end-time event is performed that is the same as the beginning one. The Wise test provides a mold gripping position $\mathrm{A}$ and a resting hand position $\mathrm{C}$. At this stage, the unit was never removed during the 10 blocks to study the repeatability of the sensors. A second stage with B in the same position of A and $\mathrm{D}$ in the same position of $\mathrm{C}$, the sensor unit was removed and worn again to study the reproducibility of the sensors. During processing, all signals start from the sample corresponding to the initial peak. With the introduction of the spline function, the error introduced in the angular values is less than 1 bit, which is negligible in the scale considered. Once synchronized and aligned, the period was segmented in 10 seconds intervals, corresponding to each position and each stimulus provided by Arduino Nano. From these 10 seconds, known the reference work frequency, the central 6 seconds on which to apply the Wise analysis were extracted. The Working matrix, defined as D, is 4-dimensional, indexed by the iteration number (containing the mean value on 6 seconds for each sensor), block number, sensor number and position number. A first code deals with the organization of data in the D matrix for each subject, whereas a second code is used to calculate the Range and standard deviation (SD) values, intra-correlation coefficients (ICCs), and correlation between Range and SD for all subjects. The bending angles of the hand joints were measured without and with wearing the data glove, using a manual goniometer with 1 degree resolution; the obtained values were used in calibration for mapping the digital values into angular values.

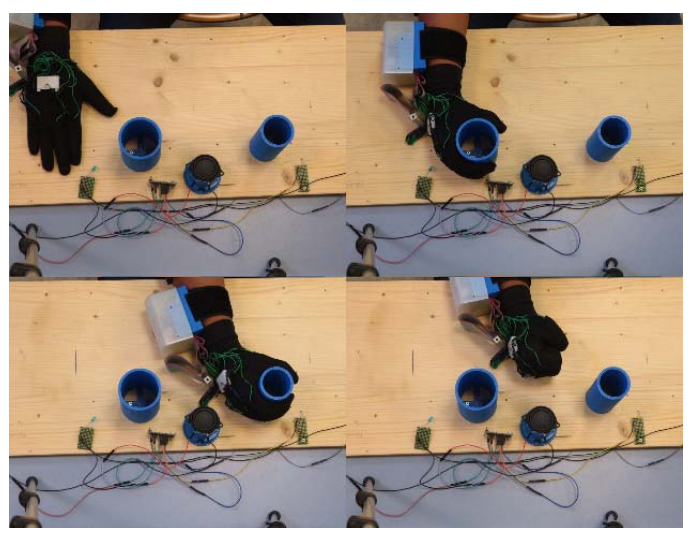

Fig. 3. Hand positioning during Wise test: 1) open hand, 2) large mold grasping 3 ) small mold grasping 4) hand completely closed.

\section{RESULTS}

The developed code provided tabular Wise-based Range and SD values for each subject and the mean of Range and $\mathrm{SD}$ values among the participants. Only the average values are shown in the present study.

Table I shows full Range and SD values for all molds. The small mold was considered the most similar to the mold used in the Wise tests.

A comparison between Range and SD values in literature for gloves based on bend sensors and inertial sensors is reported in Table II. In the upper part of the table are the values of sensory gloves based on flex sensors. In the lower part of the table the Range and SD values of gloves based on IMU sensors are also reported. For these inertial sensors, the test result $\mathrm{C}$ and $\mathrm{D}$ are not reported because it is not possible to do the doffing, since the devices have not a fabric support.

However, our table cannot be compared with other similar tables, because a repeatability study in Wise test with decreasing size molds does not exist in literature.

The correlation graphs between Range and SD values are reported for the 4 tests in the Wise protocol with reference to the big mold (Fig. 4), small mold (Fig. 5) and closed hand (Fig. 6). The standard deviation graph for each sensor is also reported. The mean SD among all subjects for each Hiteg glove sensor through the Wise test is reported in Fig. 7. 
TABLE I: COMPARISON OF RANGE AND SD VALUES FOR THE HITEG GLOVE ACCORDING TO THE MOLD USED IN THE WISE TEST.

\begin{tabular}{|c|c|c|c|c|c|c|c|c|c|c|}
\hline & \multicolumn{2}{|c|}{ Test A } & \multicolumn{2}{c|}{ Test B } & \multicolumn{2}{c|}{ Test C } & \multicolumn{2}{c|}{ Test D } & \multicolumn{2}{c|}{ Mean Value } \\
\cline { 2 - 11 } & Range & SD & Range & SD & Range & SD & Range & SD & Range & SD \\
\hline Big mold & 6.67 & 2.12 & 6.85 & 2.16 & 3.47 & 1.11 & 4.45 & 1.44 & 5.36 & 1.71 \\
\hline Small mold & 7.98 & 2.52 & 8.16 & 2.58 & 4.06 & 1.27 & 4.87 & 1.59 & 6.27 & 1.99 \\
\hline Closed hand & 8.18 & 2.60 & 8.91 & 2.92 & 4.31 & 1.14 & 3.55 & 1.40 & 6.24 & 2.01 \\
\hline
\end{tabular}

TABLE II: COMPARISON BETWEEN RANGE AND SD VALUES IN LITERATURE FOR GLOVES BASED ON BEND SENSORS AND INERTIAL SENSORS

\begin{tabular}{|c|c|c|c|c|c|c|c|c|c|c|}
\hline & \multicolumn{2}{|c|}{ Test A } & \multicolumn{2}{c|}{ Test B } & \multicolumn{2}{c|}{ Test C } & \multicolumn{2}{c|}{ Test D } & \multicolumn{2}{c|}{ Mean Value } \\
\cline { 2 - 12 } & Range & SD & Range & SD & Range & SD & Range & SD & Range & SD \\
\hline Hiteg glove & 6.67 & 2.12 & 6.85 & 2.16 & 3.47 & 1.11 & 4.45 & 1.44 & 5.36 & 1.71 \\
\hline Gentner & 6.09 & 1.94 & 7.16 & 2.26 & 2.61 & 0.86 & 3.98 & 1.28 & 4.96 & 1.59 \\
\hline Wise & 6.5 & 2.6 & 6.8 & 2.6 & 4.5 & 1.6 & 4.4 & 2.2 & 5.6 & 2.3 \\
\hline Dipietro & 7.47 & 2.44 & 9.38 & 2.96 & 3.84 & 1.23 & 5.88 & 1.92 & 6.65 & 2.14 \\
\hline Simone & 5.22 & 1.61 & & & 1 & 0.5 & & & 3.36 & 1.05 \\
\hline Kortier & 1.8 & 0.6 & & & 1.1 & 0.4 & & & 1.5 & 0.5 \\
\hline Li & 4.56 & 1.57 & & & 2.02 & 4.56 & & & 3.29 & 3.07 \\
\hline O' Flinn & 7.54 & 2.11 & & & 2.27 & 1 & & & 4.9 & 1.56 \\
\hline
\end{tabular}

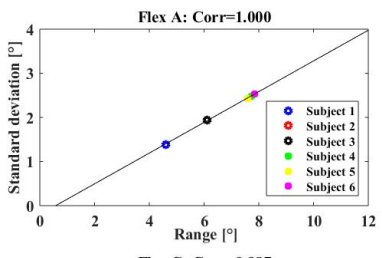

Flex C: Corr $=0.987$
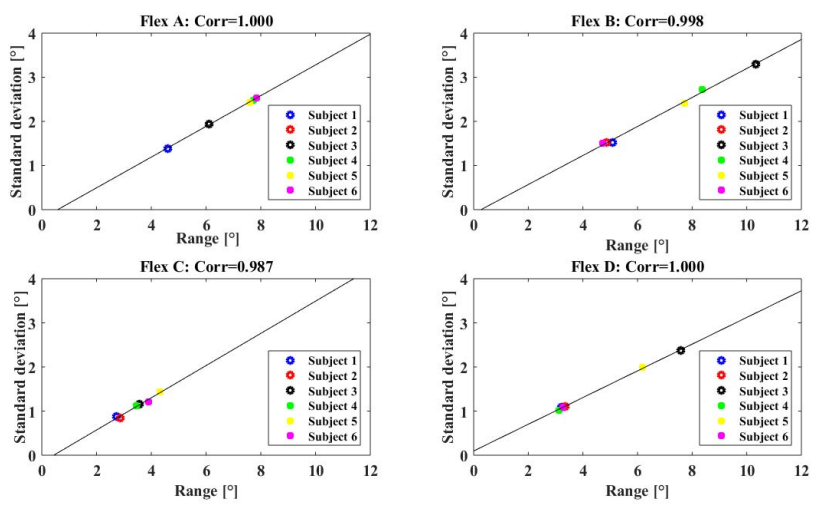

Fig. 4. Correlation values for the Hiteg glove between Range and SD of the Wise test (big mold).
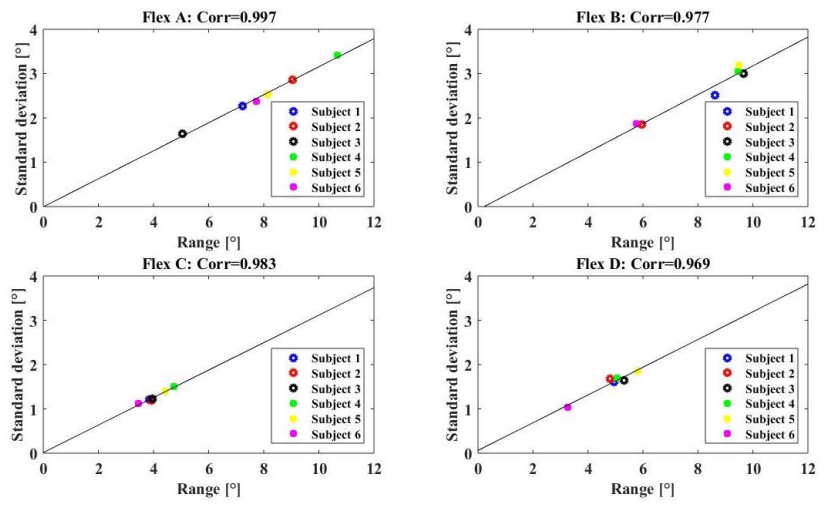

Fig.5. Correlation values for the Hiteg glove between Range and SD through the Wise test (small mold).
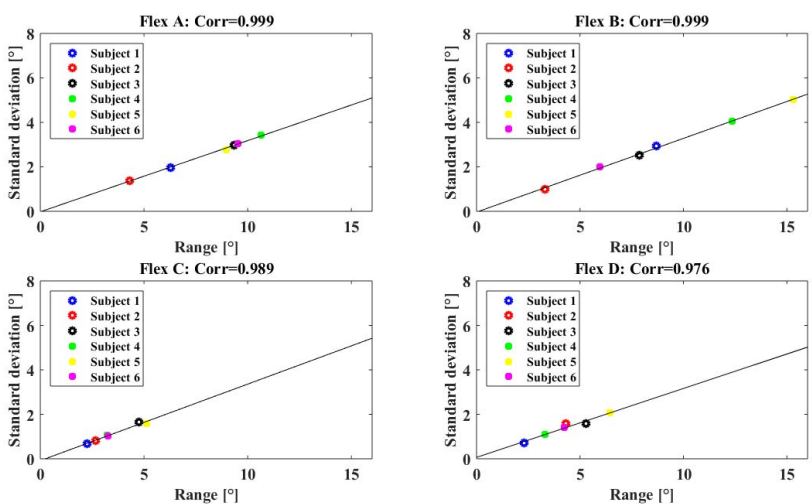

Fig.6. Correlation values for the Hiteg glove between Range and SD of the Wise test (closed hand).

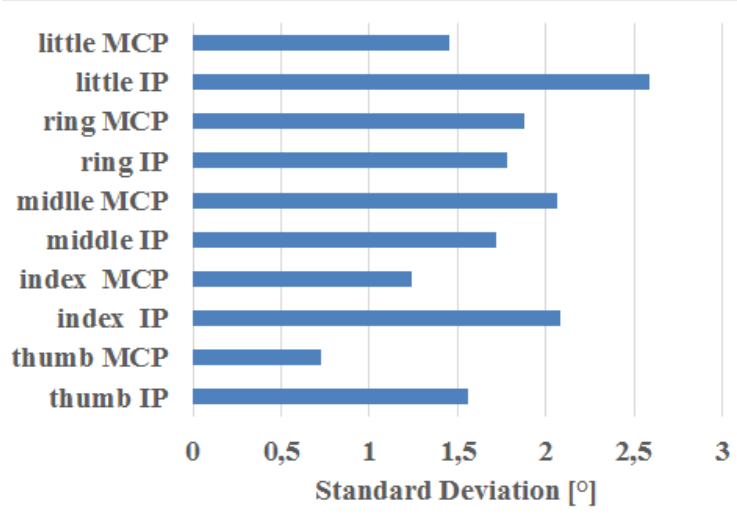

Fig.7. Mean Standard Deviation among all subjects for each Hiteg glove sensor through the Wise test. 


\section{DISCUSSION}

The mean Range values obtained are in the middle of the considered scale, lower than Dipietro [2] and Wise [6] but higher than Gentner [7] and Simone [8]. It should be noted that Simone does not provide the results for the C and D tests: if the two tests had been excluded from our protocol, it would have performed a mean value Range of 5.07 and a mean SD 1.61. The $\mathrm{C}$ and $\mathrm{D}$ tests have lower values than the corresponding $\mathrm{A}$ and $\mathrm{B}$ and this is consistent with the previous studies: placing the hand in a rest state introduces a lower error in terms of reproducibility and repeatability than the grasping of a mold which may occur from time to time with not negligible variations. The Range values from $\mathrm{B}$ to $\mathrm{A}$ and $\mathrm{D}$ to $\mathrm{C}$ are higher, and this result is also consistent: removing the glove introduces reproducibility errors. The average values for males by Range and SD are $4.70(1,50)$ lower than $6,68(2,13)$ of females: the glove best fits the male hand than the female one because the latter, while giving reasonable values during the calibration, does not fill the glove as well as a male hand. This is mainly due to the size and geometry of the hand, that varies between males and females. A result that could not be obtained from the previous studies is the variation of the Range and the SD as changing the size of the mold until it get the closed hand. Both Range and SD increase monotonically with decreasing mold size, that is with increased flexion of hand joints. This is inferred from the results in which the Range in the three studied cases is 5.36, 6.27 and 6.24 respectively. The increase in the average value of the Range is mainly due to the increase in the value of Range in the A and B tests, although with less weight in the $C$ and $D$ tests. The results are reasonable since the calibration is within the range between the minimum and the set flexion: the Range is less in the interval 0-30 than 0-60 and 0-90 degrees.

The Range-SD correlation values for the whole test are consistent with the previous studies [7] (Fig. 4 corr_A=1.000, corr $\mathrm{B}=0.998$, corr $\mathrm{C}=0.987$, corr $\mathrm{D}=1.000)$. These results indicate a linear relationship between the Range and SD and furnishes a comparable estimation of measurement repeatability. The $\mathrm{ICC}=0.75$ for the glove indicates a reasonable reliability. The ICC and correlation study were also conducted for the small mold $(\mathrm{ICC}=0.77)$ and the closed hand $(\mathrm{ICC}=0.78)$ ), and reasonable results were respectively equal to: 1) Fig.5 small mold, corr_ $A=0.997$, corr_ $B=0.977$, corr $\mathrm{C}=0.983$, corr $\mathrm{D}=0.969$; 2) Fig.6 closed hand, corr_A $=0.999$, corr_ $\bar{B}=0.999$, corr_C $=0.989$, corr_ $\mathrm{D}=0.976$ ). The negligible correlation variation and ICC indicate that the systemic error is invariant respect to the calibration.

There are no substantial differences between the average SD values (Fig. 7) except for the little IP joint: the highest SD in this case could be due to the non-optimal position of the sensor.

\section{CONCLUSIONS}

Semiautomatic measurements of the hand joints have been carried out to date with the use of the goniometric glove. This study carried out a new data glove equipped with 10 bending sensors, showing results similar to those already existing in the literature. This study also investigated the dependence of repeatability, reproducibility, reliability and correlation between Range and SD depending on the size of the mold used for the Wise test. It has been seen that the size of the mold could influences the Range and SD values. The Wise protocol was applied with two molds to allow an intermediate closure with respect to the open and the closed hand. The glove remains the main tool for detecting the flexion angles of the hand joints. Future work is to investigate new industrial and prototype bending sensors to test new improvements, to integrate flex sensors with miniature IMU placed on the hand phalanges, due to their introduction and application for the data glove, using the information from stretch sensors for small angles given their high sensitivity [11].

\section{REFERENCES}

[1] K. Li, I-M. Chen, S. H. Yeo, C. K. Lim, "Development of finger-motion capturing device based on optical linear encoder", Journal of Rehabilitation Research \& Development, Vol. 48, No. 11, pp. 68-72, 2011. http://dx.doi.org/10.1682/JRRD.2010.02.0013

[2] L. Dipietro, A. M. Sabatini, "A Survey of Glove-Based Systems and Their Applications", IEEE Trans. on Systems, Man, And CyberneticsPart C: Applications and Reviews, Vol. 38, No. 4, July 2008. http://dx.doi.org/10.1109/TSMCC.2008.923862

[3] G. Saggio, "A novel array of flex sensors for a goniometric glove", Elsevier, Sensors and Actuators A 205 (2014) 119-125, http://dx.doi.org/10.1016/j.sna.2013.10.030

[4] H. G. Kortier, V. I. Sluiter, D. R. and P. H. Veltink, "Assessment of hand kinematics with inertial and magnetic sensors", Journal of NeuroEngineering and Rehabilitation 2014, 11:70 http://www.jneuroengrehab.com/content/11/1/70

[5] B. O'Flinn, J. T. Sanchez, S. Tedesco, B. Downes, J. Connolly, J. Condell, K. Curran, "Novel Smart Glove Technology as a Biomechanical Monitoring Tool", Sensors \& Transducers, Vol. 193, Issue 10, October 2015, pp. 23-32.

http://www.sensorsportal.com/HTML/DIGEST/P_2731.htm

[6] S. Wise, W. Gardner, E. Sabelman, E. Valainis, Y. Wong, K. Glass, J. Drace, J. M. Rosen, "Evaluation of a fiber optic glove for semiautomated goniometric measurements", Journal of Rehabilitation Research and Development Vol. 27 No. 4, 1990 Pages 411-424.

[7] R. Gentner, J. Classen, "Development and evaluation of a low-cost sensor glove for assessment of human finger movements in neurophysiological settings", Journal of Neuroscience Methods 178 (2009) 138-147, http://dx.doi.org/10.1016/j.jneumeth.2008.11.005

[8] L. K. Simone, N. Sundarrajan, X. Luoc, Y. Jia, D. G. Kamper, "A low cost instrumented glove for extended monitoring and functional hand assessment", Journal of Neuroscience Methods 160 (2007) 335-348. http://dx.doi.org/10.1016/j.jneumeth.2006.09.021

[9] P. Cavallo, G. Saggio, "Conversion of Sign Language to Spoken Sentences by means of a Sensory Glove", Journal of Software, Vol 9, No 8 (2014), Aug 2014. http://dx.doi.org/10.4304/jsw.9.8.2002-2009

[10] G. Saggio, A. Pallotti, L. Sbernini, V. Errico, F. Di Paolo, "Feasibility of Commercial Resistive Sensors Flex Hand Tracking for Applications", Sensors \& Transducers, Vol. 201, Issue 6, June 2016, pp. 17-26. http://www.sensorsportal.com/HTML/DIGEST/P_2827.htm

[11] G. Saggio, S. Bocchetti, C.A. Pinto, G. Orengo, F. Giannini, "A novel application method for wearable bend sensors", 2nd International Symposium on Applied Sciences in Biomedical and Communication Technologies (ISABEL), Bratislava, Slovak Republic, 2009. http://dx.doi.org/10.1109/ISABEL.2009.5373625

[12] L. Sbernini, A. Pallotti, G. Saggio, "Evaluation of a Stretch Sensor for its Inedited Application in Tracking Hand Finger Movements", IEEE International Symposium on Medical Measurements and Applications (MEMEA 2016), May 15-18, 2016, Benevento, Italy, https://doi.org/10.1109/MeMeA.2016.7533809 